\title{
Use of Tramadol or Other Analgesics in Patients Treated in the Emergency Department as a Risk Factor for Opioid Use
}

\author{
Jorge Enrique Machado-Alba (D), ${ }^{1}$ Laura Sofía Serna-Echeverri (iD, ${ }^{1}$ \\ Luis Fernando Valladales-Restrepo $\mathbb{C D}^{1,2}$ Manuel Enrique Machado-Duque $\mathbb{D}^{1,2}$ \\ and Andrés Gaviria-Mendoza $\mathbb{D}^{1,2}$ \\ ${ }^{1}$ Grupo de Investigación en Farmacoepidemiología y Farmacovigilancia, Universidad Tecnologica de Pereira-Audifarma S.A, \\ Address: Calle 105 No. 14-140, Pereira, Risaralda, Colombia \\ ${ }^{2}$ Grupo de Investigación Biomedicina, Facultad de Medicina, Fundación Universitaria Autónoma de las Américas, \\ Address: Ave Las Americas \# 98-56, Pereira, Colombia
}

Correspondence should be addressed to Jorge Enrique Machado-Alba; machado@utp.edu.co

Received 15 August 2020; Revised 9 October 2020; Accepted 31 October 2020; Published 21 November 2020

Academic Editor: Li Hu

Copyright (c) 2020 Jorge Enrique Machado-Alba et al. This is an open access article distributed under the Creative Commons Attribution License, which permits unrestricted use, distribution, and reproduction in any medium, provided the original work is properly cited.

\begin{abstract}
The objective of this cohort study was to determine the association between the use of tramadol in emergency departments and the later consumption of opioids at the outpatient level in a group of patients from Colombia. Based on a medication dispensation database, patients over 18 years of age treated in different clinics in Colombia who for the first time received tramadol, dipyrone, or a nonsteroidal anti-inflammatory drug (NSAID) in the emergency room between January and December 2018 were identified. Three mutually exclusive cohorts were created, and each patient was followed up for 12 months after the administration of the analgesic to identify new formulations of any opioid. A Cox proportional-hazards regression model was constructed to identify variables associated with receiving a new opioid. A total of 12,783 patients were identified: 6020 treated with dipyrone, 5309 treated with NSAIDs, and 1454 treated with tramadol. The mean age was $47.1 \pm 20.4$ years, and $61.6 \%$ were women. A total of $17.3 \%(n=2207)$ of all patients received an opioid during follow-up. Those treated with tramadol received a new opioid with a higher frequency $(n=346,23.8 \%)$ than the other cohorts (14.7\% NSAIDs and 17.9\% dipyrone, both $p<0.001)$. In the tramadol group, using more than $10 \mathrm{mg}$ of morphine equivalents was associated with a greater use of new opioids (HR:1.47, 95\%CI: 1.12-1.93). Patients treated with tramadol in emergency departments have a higher risk of opioid use at the one-year follow-up than those treated with NSAIDs or dipyrone.
\end{abstract}

\section{Introduction}

Opioids are a group of drugs used mainly in the treatment of acute pain of moderate to severe intensity, as well as for the management of chronic pain, especially in cancer patients; however, they are not a first-line option in chronic noncancer pain $[1,2]$. The continuous use of these medications generates tolerance, which can lead to abuse and physical and mental dependence, in addition to an increased risk of adverse drug reactions $[3,4]$. Some estimates show that there are 15 million people in the world who are dependent on these drugs [5]. In the last two decades, the prescription of opioids has increased by $300 \%$ [6], and with it there has been an increase in cases of overdose and related deaths, due in part to their greater use in the treatment of chronic noncancer pain [5, 6]. According to the 2019 report of the United Nations Office on Drugs and Crime, it was estimated that 53.4 million people consumed opioids in $2017,56 \%$ more than in 2016. A total of $29.2 \%$ consumed heroin or opium, which was 50\% higher than the 2016 estimate [7].

On the other hand, Cicero et al. described that $47.1 \%$ of patients with substance-use disorders had their first exposure to opioids after a prescription by a doctor for pain management [8]. Numerous studies have shown that the use 
of opioids in the postoperative period increases the risk of their chronic use [5, 9-13]. Among the identified risk factors for chronic consumption of opioids are a history of alcohol or psychoactive substance abuse, current or previous smoking, comorbidities, psychiatric disorders, use of antidepressants or benzodiazepines, lower educational level, and lower income [5, 9-12]. Tramadol has been considered a moderately narcotic opioid analgesic since it is a partial agonist of $\mu$ (mu) receptors, and it has been found that it has a lower risk of dependence and other adverse reactions compared to other opioid analgesics, such as morphine, methadone, and fentanyl $[4,14,15]$. However, recently, in the USA, patients with postsurgical pain treated with tramadol were found to have a $6 \%$ greater risk of receiving an opioid again after 90 to 180 days compared to patients treated with other short-acting opioids [13].

In studies conducted in Colombia, codeine (57.7\%) and tramadol (30.9\%) were the most prescribed opioid analgesics in outpatients [16], whereas in patients with postoperative pain in eight clinics in the country, tramadol was the most commonly used (25.8\%) [17]. Given the high frequency of the use of tramadol along with the little information about the real risk of increasing the probability of using opioids again, we sought to determine the association between the administration of tramadol or other nonopioid analgesics in emergency departments and the risk of later opioid consumption at an outpatient level in Colombian patients.

\section{Materials and Methods}

A cohort study was conducted with patients seen at five clinics in Colombia between January 1 and December 31, 2018, of either sex, older than 18 years, who received tramadol, nonsteroidal anti-inflammatory drugs (NSAIDs), or dipyrone during their stay. The patients were identified from the drug dispensing database of Audifarma SA, which delivers drugs to different clinics and hospitals in the country as well as to outpatients.

Three mutually exclusive follow-up cohorts were created depending on which drug they received (at least one dose) in the emergency department:

(i) First cohort: patients who were administered tramadol orally or parenterally during their stay.

(ii) Second cohort: patients who were administered some NSAID or acetaminophen (which was included in the group of NSAIDs for their similar mechanism of action) orally or parenterally.

(iii) Third cohort: patients who were administered dipyrone orally or parenterally.

The patients of the three cohorts were over 18 years of age, of any sex, and were affiliated with any of the healthcare insurance companies to which Audifarma SA dispensed medications, so that prior and subsequent prescriptions could be identified in the database that contains information on each subject and ensures that the patient is active in the Colombian health system.
2.1. Criteria for Exclusion. Patients who received an opioid, a benzodiazepine, or an antidepressant for up to 24 months before emergency room care at their respective clinics, either at prior visits to any of these services or at the outpatient level, were excluded from the three cohorts. Also excluded were those who received combined analgesia during their stay (a combination of tramadol and any other opioid, a NSAID, acetaminophen, or dipyrone or combinations of these). Similarly, people who during the 12-month follow-up period were administered an opioid for new hospitalization were excluded.

2.2. Variables. A database was created from the information stored on the medications dispensed to these patients, and the following variables were included:

(i) Sociodemographic: age, sex, and city of residence, grouped according to geographic region of the country, distributed as follows: 1- central-western region, 2- Atlantic region, 3- Bogota and eastern region, 4- southwestern region, and 5- southern region.

(ii) Pharmacological: (a) received opioid: tramadol, morphine, fentanyl, codeine, etc.; (b) NSAIDs: diclofenac, ibuprofen, naproxen, etc.; (c) acetaminophen; and (d) dipyrone (metamizole sodium). The patients received doses even during the hospitalization beyond the stay in the emergency department). The doses were calculated according to the defined daily dose (DDD) (according to the World Health Organization, DDD is the mean dose used each day of a drug in its main indication in adults), and in the case of tramadol, its dosage was converted to morphine equivalents. Total morphine equivalents were calculated by multiplying the quantity of each prescription by the strength of the prescription (milligrams of opioid per unit dispensed). The quantity $\times$ strength product was then multiplied by the conversion factor for morphine equivalents ( 0.1 for tramadol) to estimate the morphine equivalents for the prescription.

(iii) Comorbidities (sought from the codes of the International Classification of Diseases ICD-10 at the time of emergency room care) related to painful disorders or the use of drugs with addictive potential (cancer, anxiety, psychoactive substance use, alcoholism, depression, epilepsy, schizophrenia, bipolar affective disorder, depression, diabetes mellitus, migraine, other types of headaches, rheumatoid arthritis, ankylosing spondylitis, fibromyalgia, and osteoarthritis).

2.3. Outcome. Patients from each cohort were followed up for 12 months from the date they first received tramadol or the nonopioid analgesic to identify the new formulations of any opioid medications that they had received as outpatients. The time to prescription of the opioid, the duration of 
TABLE 1: Clinical characteristics related to the prescription of tramadol, nonsteroidal anti-inflammatory drugs, and dipyrone in 12,783 patients treated in the emergency departments of five clinics in Colombia, 2018-2019.

\begin{tabular}{|c|c|c|c|c|c|}
\hline Characteristics & $\begin{array}{c}\text { Total } \\
12783 \\
\end{array}$ & $\begin{array}{c}\text { Tramadol } \\
n=1454\end{array}$ & $\begin{array}{l}\text { NSAIDs } \\
n=5309\end{array}$ & $\begin{array}{c}\text { Dipyrone } \\
n=6020\end{array}$ & $p$ \\
\hline Women & $7872(61.6 \%)$ & $828(56.9 \%)$ & $3238(61.0 \%)$ & $3806(63.2 \%)$ & $<0.001$ \\
\hline Age (years) & $47.1 \pm 20.4$ & $53.1 \pm 20.3$ & $43.5 \pm 19.5$ & $48.8 \pm 20.6$ & 0.005 \\
\hline Mean doses \pm SD (mg) & & $60.0 \pm 29.7$ & $\begin{array}{c}\text { Ds: } 75.9 \pm 10.1 \\
\text { Ac: } 2689 \pm 2339\end{array}$ & $1500 \pm 1100$ & \\
\hline Received opioid at follow-up & $2207(17.3 \%)$ & $346(23.8 \%)$ & $778(14.7 \%)$ & $1083(17.9 \%)$ & $<0.001$ \\
\hline Time to opioid in days & & $135.2 \pm 114.7$ & $143.1 \pm 106.6$ & $142.2 \pm 110.3$ & $<0.001$ \\
\hline Range of time to receive opioid (days) & $1-360$ & $1-358$ & $1-360$ & $1-360$ & \\
\hline
\end{tabular}

NSAIDs: nonsteroidal anti-inflammatory; SD: standard deviation; Ds: diclofenac sodium; Ac: acetaminophen.

use (in days), the opioid medication received, and the dose were determined.

2.4. Statistical Analysis. The statistical package SPSS 26.0 for Windows (IBM, USA) was used to analyze the data. Frequencies, proportions, means, and standard deviations were established. For the comparison of the cohorts, parametric and nonparametric tests were used according to the normality of the distribution. Kaplan-Meier type analysis was used to establish the time until the prescription of a new opioid, where $T_{o}$ was the index moment at which each patient first received the analgesic in the emergency department and $T_{k}$ was the moment at which a new opioid was received. Bivariate analyses were performed to compare the different variables (age, sex, geographic region, dipyrone, NSAIDs, and comorbidities) identified based on whether the opioid was received or not during the 12 months after emergency care, using $\mathrm{X}^{2}$ tests. Cox proportional-hazards model was performed in which the dependent variable was receiving or not receiving a new opioid, and the independent variables were those identified as associated with the bivariate analyses. In addition, a subanalysis to calculate the risk of receiving a new opioid in the following 12 months according to the initial dose of tramadol was performed, as follows: low doses (1 to $9 \mathrm{mg}$ morphine equivalents) or medium doses of tramadol ( $\geq 10 \mathrm{mg}$ morphine equivalents). $p<0.05$ was statistically significant.

2.5. Ethics Approval. The protocol was approved by the Bioethics Committee of the Universidad Tecnológica de Pereira in the risk-free research category. The ethical principles established by the Declaration of Helsinki were respected. No personal data of the patients were used.

\section{Results}

A total of 12,783 patients were identified in the emergency departments of five tertiary-care clinics in Colombia, affiliated with 11 different health insurers, who received one of the analgesics of interest. Dipyrone was the most frequent ( $n=6020,47.1 \%)$, followed by NSAIDs $(n=5309,41.5 \%)$ and tramadol $(n=1454,11.4 \%)$. The most frequent kind of dipyrone was injectable sodium at $1 \mathrm{~g} / 2 \mathrm{~mL}(n=3761,62.5 \%$ of patients went with this analgesic), followed by dipyrone associated with hyoscine butyl bromide $2.5 \mathrm{~g} / 20 \mathrm{mg}$ $(n=2257,37.5 \%)$. The most often prescribed NSAIDs were diclofenac sodium in an injectable solution of $75 \mathrm{mg} / 3 \mathrm{~mL}$ $(n=3360,63.3 \%)$ and acetaminophen $500 \mathrm{mg}$ tablets $(n=1880,35.4 \%)$. The most widely used type of tramadol was an injectable solution of $50 \mathrm{mg} / 1 \mathrm{~mL}(n=1393,95.8 \%)$. The three cohorts differed in sex and age (see Table 1).

The majority of patients received the analgesic for only one day ( $n=11,205,87.7 \%$, mode: 0 ; range: $0-18$ days). A total of $82.7 \%(n=4976)$ of the patients received dipyrone alone on the first day versus $88.9 \%(n=1292)$ who received tramadol and $92.9 \%(n=4936)$ NSAIDs, who on average received more doses of the drug (2.6 units/patient) compared with those of dipyrone (1.5 per patient) and tramadol (1.2 per patient). A total of $93.9 \%$ of the medications were prescribed by a general practitioner. The relationship between the mean dose and DDD showed that patients treated with dipyrone received 0.73 DDD, and those with diclofenac, acetaminophen, ibuprofen, and tramadol received $0.76 \mathrm{DDD}, 0.89 \mathrm{DDD}, 0.5 \mathrm{DDD}$, and $0.2 \mathrm{DDD}$, respectively.

3.1. Comorbidities. The most frequently found comorbidities were diabetes mellitus $(n=391,3.1 \%)$, osteoarthritis $(n=188,1.5 \%)$, cancer $(n=184,1,4 \%)$, other types of headaches $(n=119,0.9 \%)$, migraine $(n=108,0.8 \%)$, depression $(n=99,0.8 \%)$, anxiety $(n=97,0.8 \%)$, epilepsy $(n=84,0.7 \%)$, fibromyalgia $(n=61, n=0.5 \%)$, rheumatoid arthritis $(n=58,0.5 \%)$, schizophrenia $(n=24,0.2 \%)$, bipolar affective disorder $(n=16,0.1 \%)$, ankylosing spondylitis $(n=3,0.0 \%)$, and psychoactive substance use $(n=2,0,0 \%)$. Statistically significant differences in the bivariate analysis were found in tramadol versus dipyrone patients in terms of the frequency of suffering some comorbidities at the time of receiving care in the emergency room, especially cancer (tramadol $3.2 \%$ vs dipyrone $1.3 \% ; p=0.001)$, anxiety $(1.8 \%$ vs $0.5 \% ; p=0.001)$, epilepsy $(1.0 \%$ vs $0.5 \% ; p=0.038)$, diabetes mellitus (4.6\% vs $2.7 \%$; $p=0.001)$, and osteoarthritis $(2.1 \%$ vs $1.3 \% ; p=0.12)$. Statistically significant differences in the bivariate analysis were also found when comparing patients with tramadol versus NSAIDs in terms of the frequency of some comorbidities such as cancer (tramadol $3.2 \%$ vs NSAIDs $1.1 \%$; $p=0.001)$, anxiety $(1.8 \%$ vs $0.8 \% ; p=0.001)$, and diabetes mellitus $(4.6 \%$ vs $3.0 \%$; $p=0.003)$. 


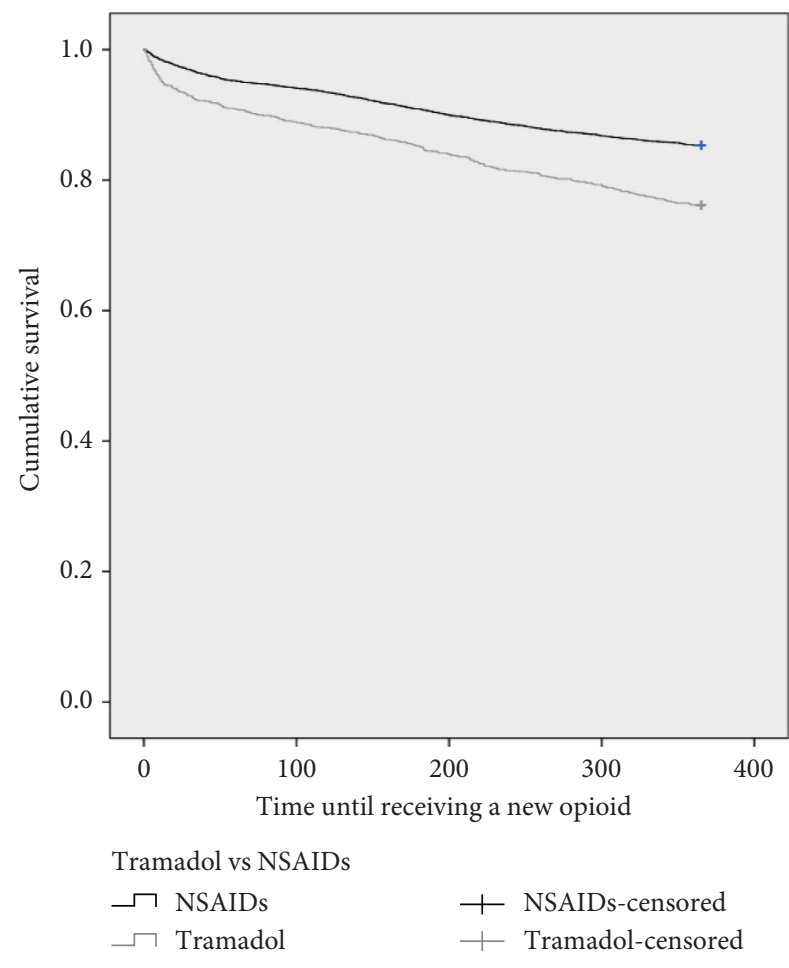

(a)

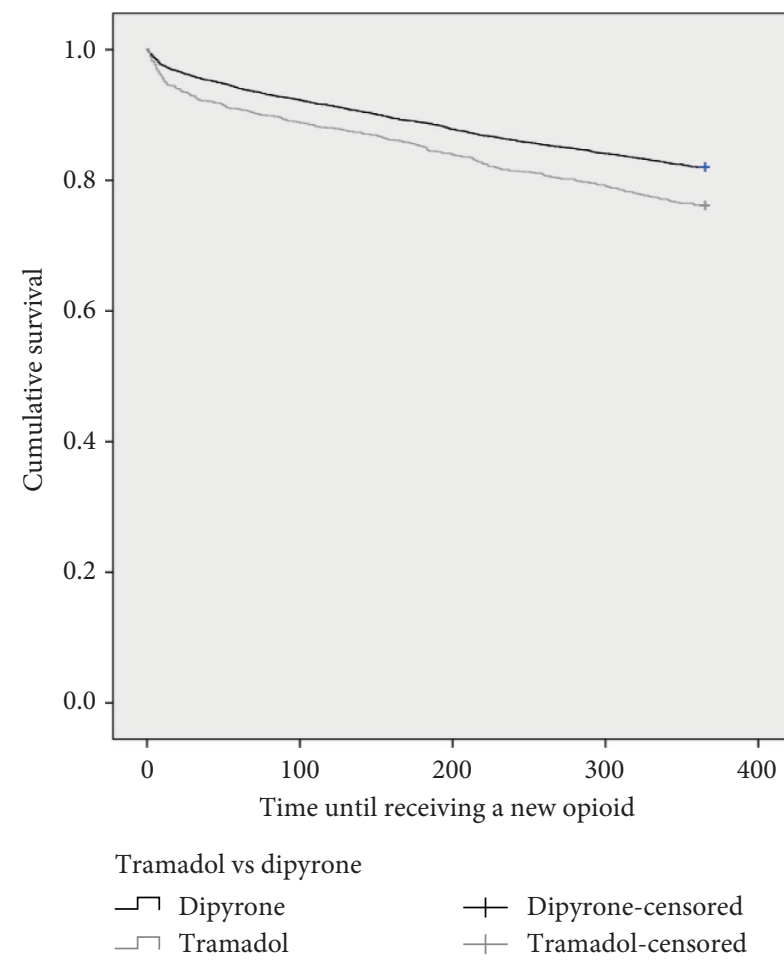

(b)

Figure 1: (a) Kaplan-Meier type analysis of the time until receiving a new opioid from the tramadol and NSAID patient cohorts at five clinics in Colombia, 2018-2019. (b) Kaplan-Meier type analysis of the time until receiving a new opioid from the tramadol and dipyrone patient cohorts at five clinics in Colombia, 2018-2019.

3.2. Use of Opioids at One-Year Follow-Up. A total of $17.3 \%$ of all patients received an opioid in the 12 months after emergency care. Patients in the tramadol cohort received a new opioid during follow-up more frequently than those in the other cohorts, and in addition, the time before receiving any opioid was significantly less (Table 1 and Figures 1(a) and $1(\mathrm{~b})$ ). Approximately $85.9 \%$ of the new opioids were prescribed by general practitioners. The most frequently used opioids in the follow-up period were acetaminophen + codeine tablet $325+8 \mathrm{mg}(n=869)$, morphine injectable solution $10 \mathrm{mg}(n=334)$, dihydrocodeine syrup $12.1 \mathrm{mg} \quad(n=283)$, fentanyl injectable solution $0.5 \mathrm{mg}$ $(n=270)$, acetaminophen + hydrocodone tablet $325+5 \mathrm{mg}$ $(n=161)$, meperidine injectable solution $100 \mathrm{mg}$, remifentanil injectable solution $2 \mathrm{mg}(n=115)$, oxycodone tablet $10 \mathrm{mg} \quad(n=26)$, methadone tablet $40 \mathrm{mg} \quad(n=8)$, and buprenorphine patch $20 \mathrm{mg}(n=6)$.

3.3. Cox Regression Comparing the Cohorts. When comparing the cohort of patients who received tramadol with each of the other two cohorts (NSAIDs and dipyrone) by Cox regression adjusted for age, sex, and geographic area, we found that those who received tramadol were more likely to receive a new opioid in the following 12 months. In addition, a higher probability to receive a new opioid was found for each year of life gained, in women, patients with cancer, diabetes mellitus, epilepsy, osteoarthritis, and in patients served in the southwestern and southern regions of the country, while those seen in Bogota and the eastern region were less likely to receive one (Figures 2 and 3).

\subsection{Analysis according to the Dose of Tramadol in Milligrams} of Morphine Equivalents. When considering the dose of tramadol initially received (expressed in milligrams of morphine equivalents), we found that receiving a dose greater than or equal to $10 \mathrm{mg}$ of morphine increased the risk of receiving an opioid again in the following 12 months (HR: 1.47; 95\%CI:1.12-1.93), as did increasing age (HR:1.01; 95\% CI:1.00-1.01) and having received the first opioid in a clinic in a region other than the central-western region of the country (HR:1.57; 95\%CI:1.23-2.00).

\section{Discussion}

The present study found an association between the use of tramadol in emergency departments and the risk of receiving an opioid again in the 12 months after its prescription compared to the use of NSAIDs, acetaminophen, or dipyrone in a group of patients aged over 18 years, affiliated with the Colombian health system. The mean age (53.1 years) of the cohort of patients who received opioids in this study was higher than that found in other studies (21.8-39.7 years) $[18,19]$, and the proportion of women (56.9\%) was higher than that of men, which is consistent with what has been reported in other studies (52.5-72.3\%) $[5,13,18-20]$. 


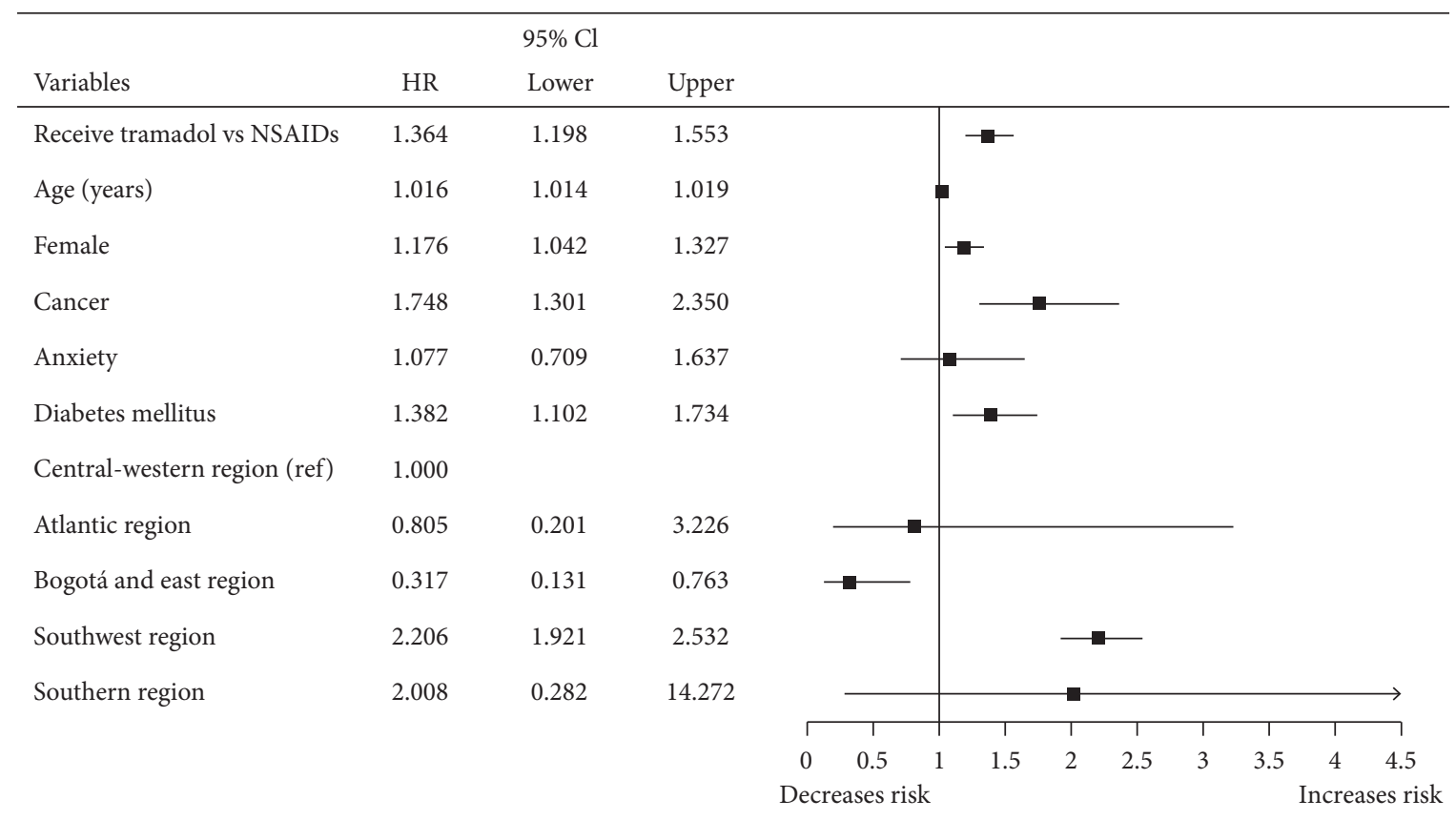

FIGURE 2: Cox regression of tramadol versus nonsteroidal anti-inflammatory drugs on the probability of receiving a new opioid up to 12 months of follow-up after being treated in emergency departments of five clinics in Colombia.

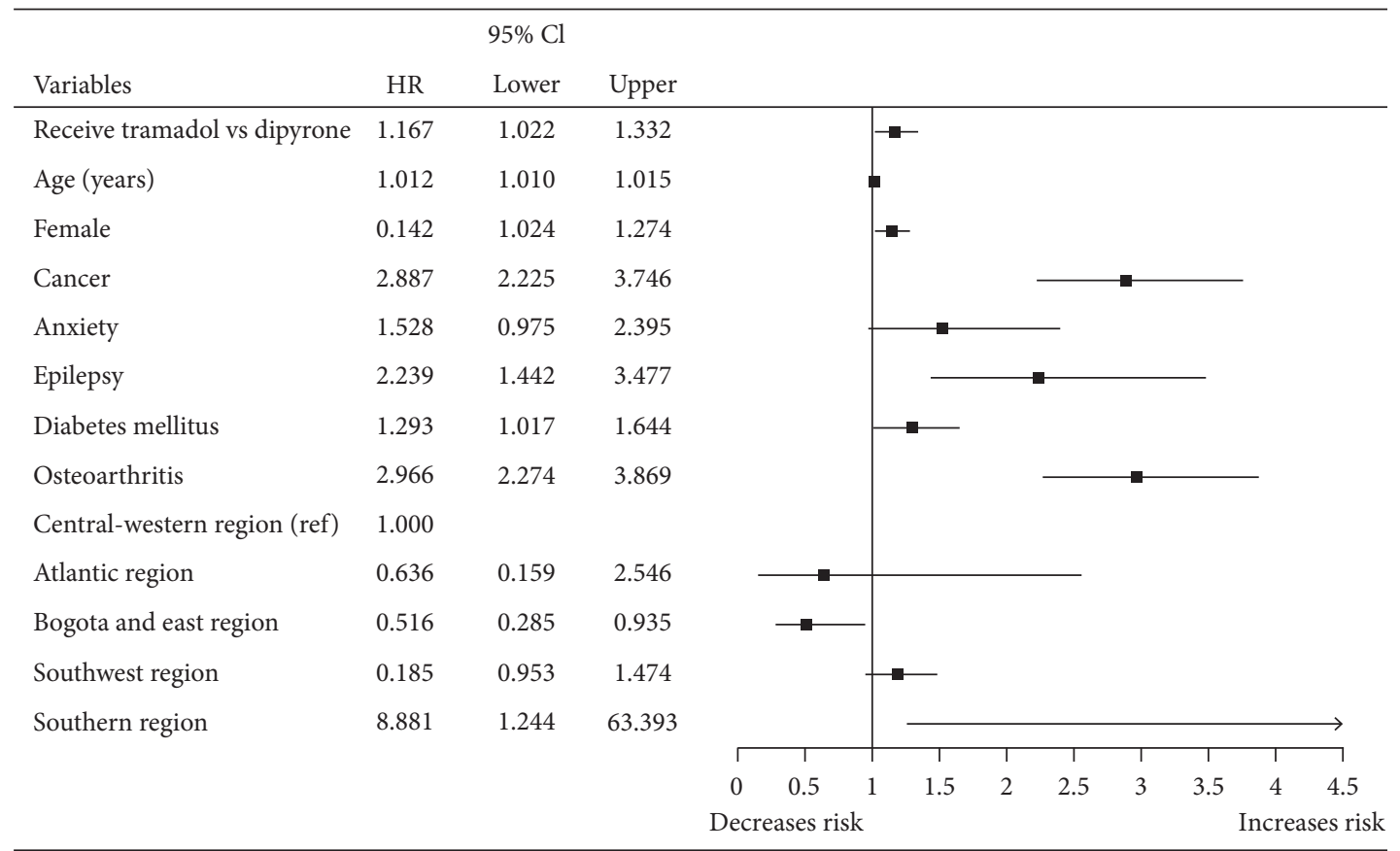

Figure 3: Cox regression of tramadol versus dipyrone on the probability of receiving a new opioid up to 12 months of follow-up after being treated in emergency departments of five clinics in Colombia, 2018-2019.

A total of $23.8 \%$ of the patients in the tramadol cohort received an opioid again in the 12 months after the initial exposure, which has also been evidenced in other studies $[5,19,21-25]$. In the USA, Hooten et al. found, in patients who had initially received an opioid who were followed up for one year, that $21 \%$ of them progressed to episodic prescriptions of opioids and $6 \%$ to continuous use [5].
Meisel et al., in patients who attended the emergency department and who were managed with opioids, found that $13.7 \%$ had persistent use [25]. Similarly, in several studies involving patients with nonobstetric postsurgical pain, frequencies of opioid use of 7.7-8.1\% were found at one year after the initial exposure $[21,22]$, while in patients who underwent a cesarean section or hysterectomy, the 
persistence of opioid use was $0.3 \%$ [23] or $0.5 \%$ [24], respectively. In patients at a dental clinic, $6.9 \%$ received a new opioid between 90 and 365 days after the initial contact [19]. These data show a worrisome reality regarding the use of opioids in emergency departments and add robustness to the findings of the present study.

In recent years, multiple studies have demonstrated that there is an increased risk of the persistent use of opioids when they are used in the management of acute pain in patients who had not previously used them $[13,18,22]$. In Canada (OR:1.44; 95\%CI:1.39-1.50) [22] and in the USA (OR:4.90; 95\%CI:3.22-7.45), the risk was significantly higher [18]. These findings are consistent with the findings in our cohort of patients. However, in some studies, the risk of receiving an opioid again after an initial exposure has been low [13], and the number of patients who may be exposed to them has been very large due to the high prevalence of use, including tramadol $[16,17,26]$, which has also increased in recent years [26].

Tramadol is a synthetic opioid, and its mechanism of action involves inhibiting the reuptake of norepinephrine and serotonin, in addition to being a partial agonist of the $\mu$ receptors $[27,28]$; therefore, it has typically been considered an analgesic with little potential for dependence, compared to other short-acting opioids [29]. However, O-desmethyltramadol, its main metabolite generated in the liver, has a 700-fold-higher affinity for the $\mu$ receptor $[27,28]$, which can help to elucidate the apparent risk if patients will use it chronically. Some authors suggest that tramadol should be reclassified [13] since it is currently in category IV of the Controlled Substances Act (Scheduled IV), which refers to drugs with a low potential for abuse and dependence [29].

Risk factors for the persistent use of opioids after the initial contact include age, sex, preoperative pain, medical comorbidities, history of substance abuse, alcohol or tobacco use, low socioeconomic status, use of benzodiazepines or antidepressants, and the dose and duration of opioid use $[7,13,19,23-25]$. We found that women were more likely to receive opioids than other drugs (13-15\% more likely than NSAIDs or dipyrone), which was consistent with, but smaller in magnitude, the data found by Thiels et al. in the USA in postsurgical patients (OR:1.22; 95\%CI:1.18-1.25) [13]. These data are also in line with those of Schroeder et al. in patients who required analgesic management in a dental clinic (OR:1.20; 95\%CI:1.00-1.40; $p=0.01$ ) [19]. The greater risk reported in women may be because they are among the population groups that most use the health services [30].

In addition, we found that the risk increased for each year of life, which was consistent with what was described by Swenson et al. in the USA (OR:2.75; 95\%CI:1.86-4.06) [24]. Likewise, an initial dose of tramadol of $10 \mathrm{mg}$ of morphine equivalents or more increased the risk of receiving an opioid as an outpatient, a trend that was also observed in other studies, but with higher tramadol doses [7, 13, 25], although other research has found no statistically significant differences [23].

The finding that cancer patients are more likely to receive opioids has already been documented, since tumors may need more effective analgesic management and they are more likely to require them at the time of emergency care, or after surgery, in the following year [31, 32]. The association found in patients with diabetes mellitus may be because they also suffer from diabetic neuropathy, which results in analgesia, paresthesia, and pain, which could yield to specific analgesics and eventually to opioids, particularly tramadol [33]. Osteoarthritis is a chronic pain disorder, which may eventually require, depending on the intensity of the symptom, analgesics capable of relieving pain when acetaminophen or NSAIDs fail to do so $[34,35]$. Furthermore, it has already been observed that epilepsy can be a predictor of subsequent opioid use, particularly in patients with a painful knee and hip disorders [35].

Certain limitations should be considered when interpreting these results. There was no access to clinical records to verify the indications for the use of the different analgesics included in the study. Other covariates that may have influenced the decision of the physician on the initial management of emergencies with analgesics were not known. For these reasons, it is important to recognize the possibility of residual confounders in the findings. In addition, it is not possible to determine if patients purchased tramadol, which was formulated outside the health system. Other variables that could be associated with the consumption of psychoactive substances by patients, as well as their tobacco use, body mass index, income level, and educational level were also not identified.

\section{Conclusions}

Based on these findings, we can conclude that patients treated with tramadol in the emergency departments of some clinics in Colombia have a higher risk of opioid use during the 12 months following their care in comparison to those given NSAIDs or dipyrone. Predominating among the higher-risk group were patients of older age, women, and those receiving tramadol doses greater than $10 \mathrm{mg}$ of morphine equivalents. Physicians should be cautious when prescribing tramadol and use it only when it is strictly necessary, and at the lowest effective doses. It is necessary to implement measures to educate prescribers about the risks of using tramadol and other opioids.

\section{Data Availability}

The data used to support the findings of the study is included within the article.

\section{Conflicts of Interest}

The authors declare no conflicts of interest.

\section{Authors' Contributions}

Jorge Enrique Machado-Alba participated in the drafting, data collection, data analysis, description of results, discussion, critical revision of the article, and evaluation of the final version of the manuscript. Laura Serna participated in the drafting, data collection, data analysis, description of results, and discussion. Manuel Enrique Machado-Duque 
participated in the drafting, data analysis, and description of results. Andres Gaviria-Mendoza and Luis ValladalesRestrepo participated in the description of results and discussion.

\section{Acknowledgments}

The authors are grateful to Carlos Tovar for his work in obtaining the database.

\section{References}

[1] C. Stein, "New concepts in opioid analgesia," Expert Opinion on Investigational Drugs, vol. 27, no. 10, pp. 765-775, 2018.

[2] D. Dowell, T. M. Haegerich, and R. Chou, "CDC guideline for prescribing opioids for chronic pain-United States, 2016," Journal of the American Medical Association, vol. 315, no. 15, pp. 1624-1645, 2016.

[3] F. Moulis, V. Rousseau, D. Abadie et al., "Effets indésirables « graves " du tramadol: bilan 2011-2015 de pharmacovigilance en France," Therapies, vol. 72, no. 6, pp. 615-624, 2017.

[4] J. J. Benich, "Opioid dependence," Primary Care: Clinics in Office Practice, vol. 38, no. 1, pp. 59-70, 2011.

[5] W. M. Hooten, J. L. St Sauver, M. E. McGree, D. J. Jacobson, and D. O. Warner, "Incidence and risk factors for progression from short-term to episodic or long-term opioid prescribing," Mayo Clinic Proceedings, vol. 90, no. 7, pp. 850-856, 2015.

[6] B. K. Madras, "The surge of opioid use, addiction, and overdoses," JAMA Psychiatry, vol. 74, no. 5, pp. 441-442, 2017.

[7] Z. Durand, S. Nechuta, S. Krishnaswami, E. L. Hurwitz, and M. McPheeters, "Prevalence and risk factors associated with long-term opioid use after injury among previously opioidfree workers," JAMA Network Open, vol. 2, no. 7, p. e197222, 2019.

[8] T. J. Cicero, M. S. Ellis, and Z. A. Kasper, "Psychoactive substance use prior to the development of iatrogenic opioid abuse: a descriptive analysis of treatment-seeking opioid abusers," Addictive Behaviors, vol. 65, pp. 242-244, 2017.

[9] E. C. Sun, B. D. Darnall, L. C. Baker, and S. Mackey, "Incidence of and risk factors for chronic opioid use among opioidnaive patients in the postoperative period," JAMA Internal Medicine, vol. 176, no. 9, pp. 1286-1293, 2016.

[10] H. Clarke, N. Soneji, D. T. Ko, L. Yun, and D. N. Wijeysundera, "Rates and risk factors for prolonged opioid use after major surgery: population based cohort study," British Medical Journal, vol. 348, no. feb11 3, p. g1251, 2014.

[11] X. Jiang, M. Orton, R. Feng et al., "Chronic opioid usage in surgical patients in a large academic center," Annals of Surgery, vol. 265, no. 4, pp. 722-727, 2017.

[12] C. M. Brummett, J. F. Waljee, J. Goesling et al., "New persistent opioid use after minor and major surgical procedures in US adults," JAMA Surgery, vol. 152, p. e170504, 2017.

[13] C. A. Thiels, E. B. Habermann, W. M. Hooten, and M. M. Jeffery, "Chronic use of tramadol after acute pain episode: cohort study," British Medical Journal, vol. 365, p. 11849, 2019.

[14] R. N. Jamison and J. Mao, "Opioid analgesics," Mayo Clinic Proceedings, vol. 90, no. 7, pp. 957-968, 2015.

[15] M. Subedi, S. Bajaj, M. S. Kumar, and M. Yc, "An overview of tramadol and its usage in pain management and future perspective," Biomedicine \& Pharmacotherapy, vol. 111, pp. 443-451, 2019.
[16] J. E. Machado-Alba, A. Gaviria-Mendoza, C. A. VargasMosquera, A. F. Gil-Restrepo, and L. C. Romero-Zapata, "Opioid prescribing patterns and costs in a large group of patients in Colombia," Journal of Pain \& Palliative Care Pharmacotherapy, vol. 31, no. 1, pp. 57-65, 2017.

[17] J. E. Machado-Alba, J. O. Ramírez-Sarmiento, and D. F. Salazar-Ocampo, "Multicenter study on effectiveness of controlling postoperative pain in Colombian patientsఓ Colombian Journal of Anesthesiology, vol. 44, no. 2, pp. 114120, 2016.

[18] S. L. Calcaterra, T. E. Yamashita, S.-J. Min, A. Keniston, J. W. Frank, and I. A. Binswanger, "Opioid prescribing at hospital discharge contributes to chronic opioid use," Journal of General Internal Medicine, vol. 31, no. 5, pp. 478-485, 2016.

[19] A. R. Schroeder, M. Dehghan, T. B. Newman, J. P. Bentley, and K. T. Park, "Association of opioid prescriptions from dental clinicians for US adolescents and young adults with subsequent opioid use and abuse," JAMA Internal Medicine, vol. 179, no. 2, pp. 145-152, 2019.

[20] N. Stark, S. Kerr, and J. Stevens, "Prevalence and predictors of persistent post-surgical opioid use: a prospective observational cohort study," Anaesthesia and Intensive Care, vol. 45, no. 6, pp. 700-706, 2017.

[21] M. A. Raebel, S. R. Newcomer, E. A. Bayliss et al., "Chronic opioid use emerging after bariatric surgery," Pharmacoepidemiology and Drug Safety, vol. 23, no. 12, pp. 1247-1257, 2014.

[22] A. Alam, T. Gomes, H. Zheng, M. M. Mamdani, D. N. Juurlink, and C. M. Bell, "Long-term analgesic use after low-risk surgery," Archives of Internal Medicine, vol. 172, no. 5, pp. 425-430, 2012.

[23] B. T. Bateman, J. M. Franklin, K. Bykov et al., "Persistent opioid use following cesarean delivery: patterns and predictors among opioid-naïe women," American Journal of $\mathrm{Ob}$ stetrics and Gynecology, vol. 215, no. 3, pp. 353.e1-353.e18, 2016.

[24] C. W. Swenson, N. S. Kamdar, K. Seiler, D. M. Morgan, P. Lin, and S. As-Sanie, "Definition development and prevalence of new persistent opioid use following hysterectomy," American Journal of Obstetrics and Gynecology, vol. 219, pp. 486.e1486.e7, 2018.

[25] Z. F. Meisel, N. Lupulescu-Mann, C. J. Charlesworth, H. Kim, and B. C. Sun, "Conversion to persistent or high-risk opioid use after a new prescription from the emergency department: evidence from Washington Medicaid beneficiaries," Annals of Emergency Medicine, vol. 74, no. 5, pp. 611-621, 2019.

[26] L. M. Bigal, K. Bibeau, and S. Dunbar, "Tramadol prescription over a 4-year period in the USA," Current Pain and Headache Reports, vol. 23, p. 76, 2019.

[27] L. Bravo, J. A. Mico, and E. Berrocoso, "Discovery and development of tramadol for the treatment of pain," Expert Opinion on Drug Discovery, vol. 12, no. 12, pp. 1281-1291, 2017.

[28] K. Miotto, A. K. Cho, M. A. Khalil, K. Blanco, J. D. Sasaki, and R. Rawson, "Trends in tramadol," Anesthesia \& Analgesia, vol. 124, no. 1, pp. 44-51, 2017.

[29] M. Gabay, "The federal controlled substances act: schedules and pharmacy registration," Hospital Pharmacy, vol. 48, no. 6, pp. 473-474, 2013.

[30] R. Mendoza-Sassi and J. U. Béria, "Utilización de los servicios de salud: una revisión sistemática sobre los factores relacionados," Cadernos de Saúde Pública, vol. 17, no. 4, pp. 819-832, 2001. 
[31] T. C. Chen, T. C. Wang, C. P. Lin et al., "Increasing tramadol utilisation under strict regulatory control of opioid prescribing-a cross-sectional study in Taiwan from 2002 through 2016," Journal of the Formosan Medical Association, vol. S0929-6646, no. 20, pp. 30452-30456, 2020.

[32] M. Falcone, C. Luo, Y. Chen et al., "Risk of persistent opioid use following major surgery in matched samples of patients with and without cancer," Cancer Epidemiology, Biomarkers \& Prevention, vol. 29, no. 11, 2020.

[33] I. Urits, N. Li, K. Berardino et al., "The use of antineuropathic medications for the treatment of chronic pain," Best Practice \& Research Clinical Anaesthesiology, vol. 34, no. 3, pp. 493506, 2020

[34] T. J. Rajamäki, P. A. Puolakka, A. Hietaharju, T. Moilanen, and E. Jämsen, "Predictors of the use of analgesic drugs 1 year after joint replacement: a single-center analysis of 13,000 hip and knee replacements," Arthritis Research and Therapy, vol. 22, no. 1, p. 89, 2020.

[35] Y. Pryymachenko, R. A. Wilson, J. H. Abbott, M. M. Dowsey, and P. F. M. Choong, "Risk factors for chronic opioid use following hip and knee arthroplasty: evidence from New Zealand population data," Journal of Arthroplasty, vol. 35, no. 11, pp. 3099-3107, 2020. 